An enamine controlling group for rhodium-

Leave this area blank for abstract info.

catalyzed intermolecular hydroacylation

Robert N. Straker, Michele Formica, James D. Lupton, Jingze Niu, and Michael C. Willis

Department of Chemistry, University of Oxford, Chemistry Research Laboratory, Mansfield Road, Oxford, OX1 3TA, UK

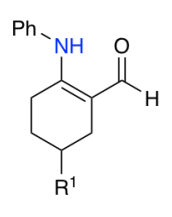

enamines

novel directing group

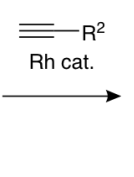

- $\mathrm{R}^{2}$<smiles>[R]C=CC(=O)C1=C(N[OH2+])CCC([Z17])C1</smiles>

$\mathrm{R}^{1}$

$\beta$-aminoenones synthetically versatile

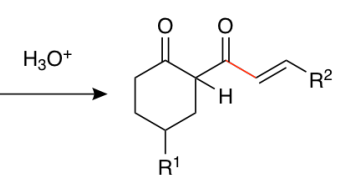

1,3-dicarbonyls $\alpha$-mono-substituted 


\title{
An enamine controlling group for rhodium-catalyzed intermolecular hydroacylation
}

\author{
Robert N. Straker, Michele Formica, James D. Lupton, Jingze Niu, and Michael C. Willis* \\ Department of Chemistry, University of Oxford, Chemistry Research Laboratory, Mansfield Road, Oxford, OX1 3TA, UK.
}

\section{ARTICLE INFO}

\section{ABSTRACT}

\section{Article history:}

Received

Received in revised form

Accepted

Available online

Keywords:

Hydroacylation

Catalysis

Heterocycle

Rhodium

\begin{abstract}
An enamine-controlled hydroacylation of alkynes using a rhodium(I)/dppe catalyst system is described. The reaction is highly selective, forming the linear enaminone products as single regioisomers in all examples. In situ hydrolysis of the enamine functionality generated $\alpha$ substituted 1,3-diketone products, and Lewis-acid mediated intramolecular conjugate addition of the hydroacylation products gave substituted hexahydroquinolones.
\end{abstract}

2018 Elsevier Ltd. All rights reserved.

\section{Introduction}

The discovery and optimization of atom efficient processes with which to construct functionalized organic scaffolds is at the forefront of modern Organic and Medicinal Chemistry. ${ }^{1,2}$ The field of hydroacylation chemistry, the formal addition of a $\mathrm{C}-\mathrm{H}$ bond across a $\pi$-bond, has grown over the past decade to not only become a robust method for the formation of ketones, enones, and esters, ${ }^{3,4}$ but also a platform for the formation of heterocycles. ${ }^{5-7}$ In the majority of cases, hydroacylation requires the use of a tethered directing group on either the aldehyde or alkene/alkyne coupling partner in order to avoid reductive decarbonylation. ${ }^{8-17}$ Recent methodologies have enabled the use of a variety of functionalities as directing groups (Scheme 1), including but not limited to; phenols, ${ }^{18-21}$ thioethers, ${ }^{22-26}$ anilines, ${ }^{27}$ and most recently, carbonyl groups. ${ }^{28}$ A limitation of ester and ketonedirected hydroacylation is that the inherent acidity of the $\alpha$-proton results in ready tautomerization under the reaction conditions, and this predominant enol form binds unproductively to the metal catalyst. This issue was alleviated by the use of $\alpha, \alpha$-disubstituted $\beta$-keto aldehydes and $\beta$-formyl esters, but $\alpha$-mono-substituted substrates could not be used. Enamines have thus far not been explored as directing groups in hydroacylation reactions, yet they have the potential not only to form synthetically useful enaminones, ${ }^{29-32}$ but also to act as carbonyl surrogates which, upon hydrolysis, would provide access to otherwise inaccessible dicarbonyl hydroacylation products. Our laboratory has previously reported amine-directed hydroacylation. ${ }^{33}$ In these reactions, the Lewis-basicity of the amine was tempered by virtue of its phenyl substituents. We envisaged aniline-derived enamines exhibiting similar reactivity in controlling hydroacylation, but with the potential for further functionalization.

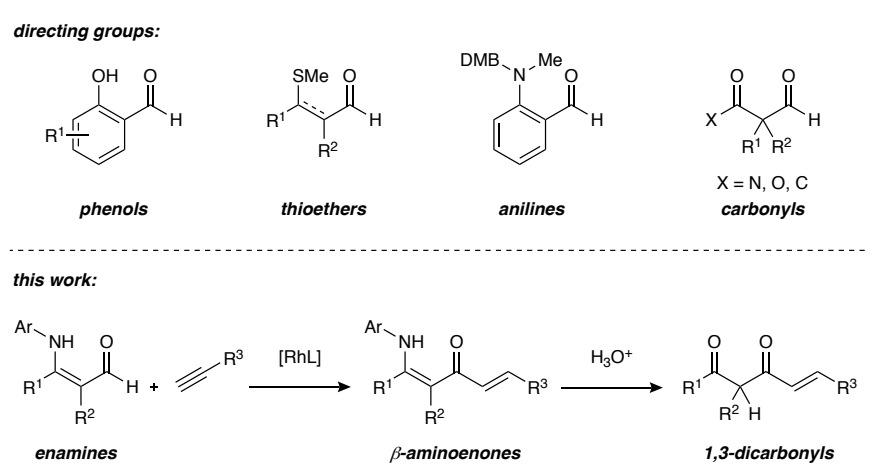

Scheme 1. Directing groups in intermolecular hydroacylation, and enamine-directed alkyne hydroacylation.

\section{Results and discussion}

After a brief evaluation of amine substituents, we chose to proceed in our investigations with cyclohexene $\beta$-aminoenal 1a, which, in combination with 1-octyne, was submitted to rhodium(I) catalysis with a number of bis-phosphine ligands of varying biteangle and phosphine substituent (Table 1). Catalyst systems comprised of narrow bite-angle ligands dcpm, dppm and PNP(Cy) led to low conversions of aldehyde substrate after 18 hours at 55 ${ }^{\circ} \mathrm{C}$, but gave high levels of regioselectivity for the linear product 2a over the branched 3a (Entries 1-3). Wider bite-angle ligands dcpe and dppe enhanced this selectivity and increased reactivity (Entries 4 and 5), with dppe generating the product in $>20: 1 \mathrm{rr}$ and in $87 \%{ }^{1} \mathrm{H}$ NMR yield. It was possible to isolate product $\mathbf{2 a}$, after purification on silica gel, as a single regioisomer in $73 \%$ yield, but 
Table 1. Optimization of enamine-directed hydroacylation reaction conditions. ${ }^{\mathrm{a}}$

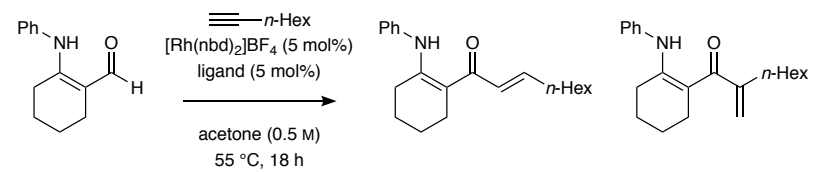

1

\begin{tabular}{llll}
\hline Entry & Ligand & Ratio $(\mathbf{2 a : 3 a})^{\mathrm{b}}$ & Yield $/ \%^{\mathrm{b}}$ \\
\hline 1 & dcpm & $9: 1$ & 40 \\
2 & dppm & $9: 1$ & 15 \\
3 & PNP(Cy) & $15: 1$ & 23 \\
4 & dcpe & $>20: 1$ & 36 \\
5 & dppe & $>20: 1$ & $87(73)^{\mathrm{c}}$ \\
6 & dppp & $>20: 1$ & 53 \\
7 & DPEphos & $9: 1$ & 7
\end{tabular}

$\begin{array}{llll}d c p m, R=C y & P N P(C y) & \text { dcpe, } R=C y & \text { dppp } \\ \text { dppm, } R=P h & & \text { DPEphos }\end{array}$

a. Reaction conditions: $\mathrm{Rh}(\mathrm{nbd})_{2} \mathrm{BF}_{4}(5 \mathrm{~mol} \%)$, ligand $(5 \mathrm{~mol} \%)$, aldehyde $(0.3$ mmol, 1.0 equiv.), alkyne (1.5 equiv.), acetone $(0.5 \mathrm{M}), 55^{\circ} \mathrm{C}$ for $18 \mathrm{~h}$. b. Yield determined by ${ }^{1} \mathrm{H}$ NMR spectroscopic analysis of the crude reaction mixture, using 1,3,5-trimethoxybenzene as internal standard. c. Isolated yield of $\mathbf{2 a}$.

it was found to hydrolyze to the 1,3-dicarbonyl product if exposed to silica for extended periods of time. Further increases to ligand bite-angle proved to be deleterious, with dppp and DPEphos both leading to reduction in reaction efficiency.

With an efficient catalytic manifold for enamine-directed hydroacylation in hand, we applied the optimized conditions to $\beta$ aminoenal 1a in reaction with various alkynes (Table 2). Pleasingly, more sterically encumbered aliphatic alkynes were well tolerated; cyclohexyl and tert-butyl substituted alkynes delivered the desired enone products $\mathbf{2 b}$ and $\mathbf{2 d}$ in $77 \%$ and $85 \%$ yield respectively. In order to demonstrate the practicability of this methodology, reaction of 1a with 3,3-dimethyl-1-butyne was performed on a $4 \mathrm{mmol}$ scale, using only $2.5 \mathrm{~mol} \%$ catalyst, which gave product $2 \mathbf{d}$ in $87 \%$ yield $(0.99 \mathrm{~g})$ after 18 hours at room temperature. Primary alkyl halides and silyl ether functionalities remained intact, with the corresponding products $\mathbf{2 e}$ and $\mathbf{2 f}$ isolated in high yields. It was found that hydroacylation of aryl alkynes was not possible under the reaction conditions, and that the aldehyde starting material could be cleanly recovered from reactions with both electron-rich and electron-poor phenylsubstituted alkynes. However, phenyl-substituted aliphatic alkyne underwent the desired hydroacylation, generating the product $\mathbf{2 g}$ in $46 \%$ yield, and phthalimide product $2 \mathbf{i}$ could also be obtained in moderate yield. We next examined variation of the aldehyde component in reaction with 1-octyne. Deviation of ring size to 5or 7-membered rings resulted in complete loss of reactivity, which is likely due to a subtle electronic effect of the cyclohexene $\beta$ aminoenal, which is disrupted by changes in geometry. Likewise, substitution to the cyclohexene ring was unfavorable and only tolerated at the 4-position. Reaction of $\mathbf{1 b}$-c required $10 \mathrm{~mol} \%$ catalyst loading, but nevertheless delivered products $\mathbf{2 j} \mathbf{j} \mathbf{- 1}$ in good yields. Complete linear regioselectivity $(>20: 1 \mathrm{rr})$ was observed in all cases, and low yielding examples were only as a result of incomplete consumption of aldehyde starting material.
Table 2. Scope of $\beta$-Aminoenal hydroacylation. ${ }^{\mathrm{a}}$
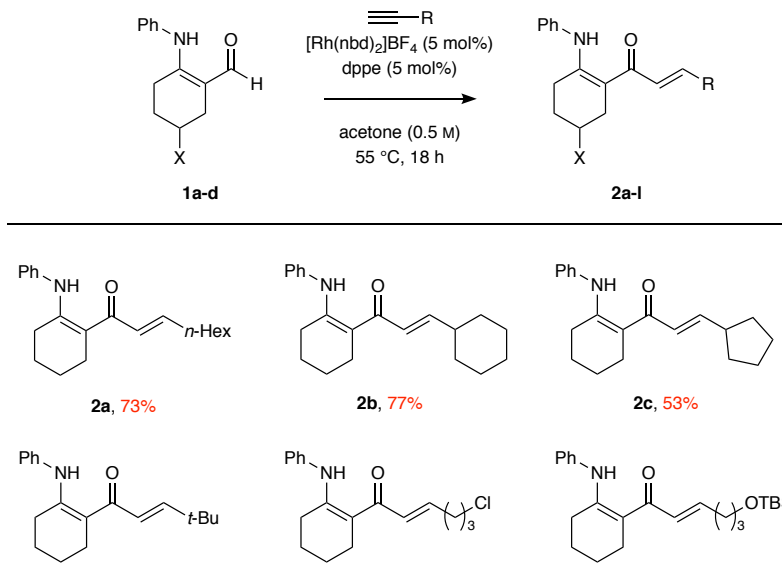

2b, $77 \%$

2c, $53 \%$
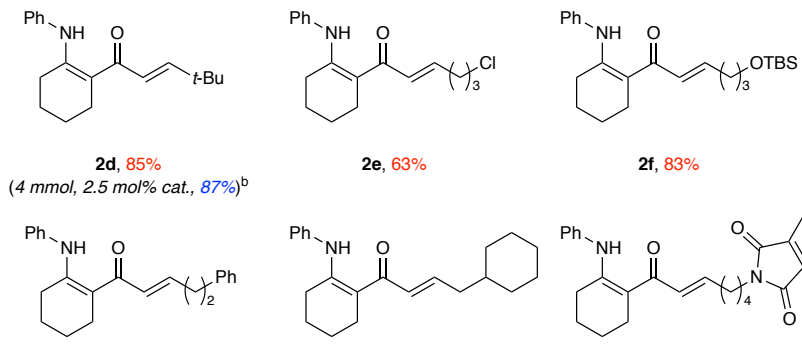

$\mathbf{2 e}, 63 \%$

2f, $83 \%$

$2 g, 46 \%$
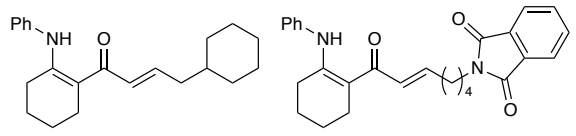

2h, $62 \%$

2i, $50 \%$

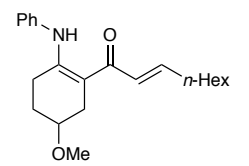

2j, $71 \%$ c

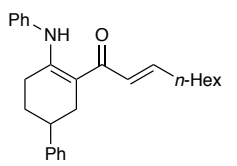

$2 \mathbf{k}, 74 \%^{\mathrm{c}}$

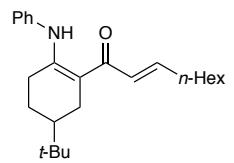

$21,66 \%^{\circ}$ a. Reaction conditions: $\mathrm{Rh}(\mathrm{nbd})_{2} \mathrm{BF}_{4}(5 \mathrm{~mol} \%)$, dppe (5 mol\%), aldehyde $(0.3$ mmol, 1.0 equiv.), alkyne ( 1.5 equiv. $)$, acetone $(0.5 \mathrm{M}), 55^{\circ} \mathrm{C}$ for $18 \mathrm{~h}$. b. Performed with $4 \mathrm{mmol}$ of aldehyde, using $2.5 \mathrm{~mol} \%$ catalyst. c. $10 \mathrm{~mol} \%$ catalyst was used.

Having developed the enamine-directed hydroacylation methodology and applied it to a number of combinations of aldehydes and alkynes, we next turned our attention to isolating the hydrolyzed enamine products from a one-pot hydroacylation/hydrolysis process (Table 3). It was found that direct addition of aqueous hydrochloric acid to the reaction vessel, following successful hydroacylation, facilitated rapid enamine hydrolysis ( $c a 2$ hours). The $\alpha$-mono-substituted 1,3-dicarbonyl products $\mathbf{4 a - h}$ were generated in good to excellent yields and were

Table 3. Tandem enamine-directed hydroacylation/hydrolysis. ${ }^{a}$

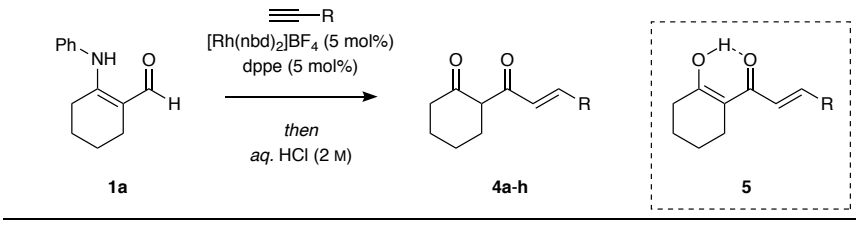

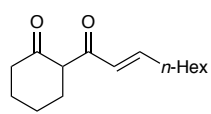

4a, $56 \%$

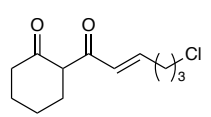

$4 \mathbf{e}, 70 \%$<smiles>O=C1CCCCC1C=CC1CCCCC1</smiles>

4b, $66 \%$<smiles>O=C(/C=C/Nc1ccccc1)C1CCCCC1=O</smiles>

$4 \mathrm{~g}, 49 \%$<smiles>CC(C)(C)C=CC(=O)C1CCCCC1=O</smiles>

4d, $88 \%$

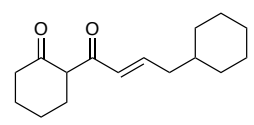

4h, $73 \%$ a. Reaction conditions: $\mathrm{Rh}(\mathrm{nbd})_{2} \mathrm{BF}_{4}(5 \mathrm{~mol} \%)$, dppe ( $\left.5 \mathrm{~mol} \%\right)$, aldehyde (0.3 mmol, 1.0 equiv.), alkyne ( 1.5 equiv.), acetone $(0.5 \mathrm{M}), 55^{\circ} \mathrm{C}$ for $18 \mathrm{~h}$, then aq. $\mathrm{HCl}(2 \mathrm{M}, 0.6 \mathrm{~mL})$. 
indeed found to exist entirely as the enol tautomer (5), as was observed by ${ }^{1} \mathrm{H}$ NMR spectroscopy in $\mathrm{CDCl}_{3}$, and confirmed by HMBC analysis.

Finally, we investigated the possibility of further utilizing the enamine functionality in a Lewis acid-catalyzed aza-conjugate addition process ${ }^{34}$ in order to construct functionalized hexahydroquinolinones (Table 4). Pleasingly, using catalytic antimony(III) trichloride in acetonitrile for 18 hours at $55{ }^{\circ} \mathrm{C}$, the isolated hydroacylation products (2) underwent the desired intramolecular cyclisation to generate bicyclic products $\mathbf{6 a - c}$ in high yields. In the case of $\mathbf{6 b}$ and $\mathbf{6 c}$, which bore substituents on the cyclohexene ring, low diastereoselectivity was observed in the conjugate addition (1.5:1 dr and 1.8:1 dr respectively). This was perhaps due to the planarity of the enamine preventing effective chirality relay in the formation of the new stereocentre.

Table 4. Lewis acid-mediated aza-conjugate addition. ${ }^{a}$
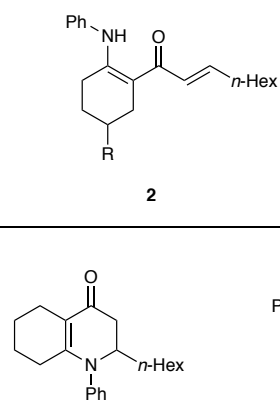

6a, $73 \%$

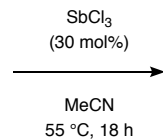

$55^{\circ} \mathrm{C}, 18 \mathrm{~h}$

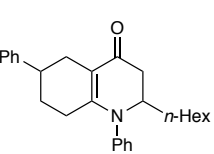

6b, $85 \%, 1.5: 1 \mathrm{dr}^{\text {b }}$

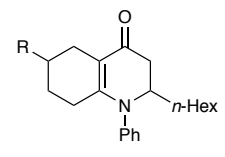

6

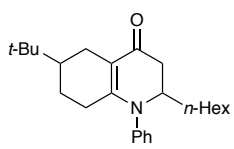

6c, $77 \%, 1.8: 1 \mathrm{dr}$ b a. Reaction conditions: $\beta$-aminoenone ( $0.2 \mathrm{mmol}, 1.0$ equiv.), $\mathrm{SbCl}_{3}$ (30 $\operatorname{mol} \%), \operatorname{MeCN}(0.3 \mathrm{M}), 55{ }^{\circ} \mathrm{C}$ for $18 \mathrm{~h}$. b. Diastereomeric ratio determined by ${ }^{1} \mathrm{H}$ NMR spectroscopic analysis of the crude reaction mixture.

\section{Conclusions}

We have demonstrated enamines as efficient directing groups for intermolecular hydroacylation of alkynes. Using a rhodium(I)/ dppe catalyst system, the enaminone products were generated as single regioisomers $(>20: 1 \mathrm{rr})$, and isolated in moderate to high yields. These products could alternatively be hydrolyzed in situ to access the corresponding 1,3-dicarbonyl products, previously inaccessible using carbonyl-directed hydoacylation, or cyclized via intramolecular aza-conjugate addition to generate synthetically attractive hetero-bicyclic systems.

\section{Experimental}

\subsection{General information}

All reactions were performed under argon using standard Schlenk techniques. ${ }^{1} \mathrm{H}$ and ${ }^{13} \mathrm{C}$ NMR spectra were obtained on a Brüker AVIII400 (400 MHz) spectrometer using the residual sovent signal as an internal standard $\left(\mathrm{CDCl}_{3}: \delta_{\mathrm{H}}=7.26 \mathrm{ppm}, \delta_{\mathrm{C}}=\right.$ $77.16 \mathrm{ppm})$. All coupling constants ( $J$ values) were reported in Hertz (Hz). Multiplicities were reported as follows: s, singlet; $d$, doublet; $\mathrm{t}$, triplet; q, quartet; quin., quintet; m, multiplet. Highresolution mass spectrometry (HRMS) measurements were recorded on a Bruker Daltronics microTOF (ESI) spectrometer. Infrared spectra were recorded as thin films on a Bruker Tensor 27 FT-IR spectrometer. Flash chromatography was carried out using matrix 60 silica. All alkynes were distilled prior to use. Acetone was dried over Drierite ${ }^{\mathrm{TM}}$ overnight, distilled at atmospheric pressure, and degassed with argon prior to use.

\subsection{Rhodium-catalyzed $\beta$-aminoenal hydroacylation}

\subsubsection{General procedure; $0.3 \mathrm{mmol}$ scale}

A $10 \mathrm{~mL}$ microwave vial containing $\left[\mathrm{Rh}(\mathrm{nbd})_{2}\right] \mathrm{BF}_{4}(5 \mathrm{~mol} \%)$ and dppe $(5 \mathrm{~mol} \%)$ was placed under vacuum $(0.05 \mathrm{mbar})$ and back-filled with argon. This vacuum cycle was repeated three times. The mixture was dissolved in acetone $(0.5 \mathrm{~mL})$, and hydrogen bubbled through the solution for $1 \mathrm{~min}$. The solvent was then removed by purging the vial with argon. The air and moisture sensitive catalyst residue was dissolved in acetone $(0.6 \mathrm{~mL})$, and the solution added to an argon-filled $10 \mathrm{~mL}$ microwave vial containing aldehyde ( $0.3 \mathrm{mmol}, 1.0$ equiv.) and alkyne $(0.45$ mmol, 1.5 equiv.). The solution was stirred at $55^{\circ} \mathrm{C}$ for $18 \mathrm{~h}$, after which time the reaction mixture was concentrated in vacuo. The crude material was purified by flash column chromatography.

\subsubsection{General procedure; 4 mmol scale}

A $10 \mathrm{~mL}$ microwave vial containing [Rh(nbd)2]BF4 (2.5 mol\%) and dppe $(2.5 \mathrm{~mol} \%)$ was placed under vacuum $(0.05$ mbar) and back-filled with argon. This vacuum cycle was repeated three times. The mixture was dissolved in acetone $(2.0 \mathrm{~mL})$, and hydrogen bubbled through the solution for $2 \mathrm{~min}$. The solvent was then removed by purging the vial with argon. The air and moisture sensitive catalyst residue was dissolved in acetone $(4.0 \mathrm{~mL})$, and the solution added to an argon-filled $25 \mathrm{~mL}$ round-bottom flask containing a stirred solution of aldehyde ( $4.00 \mathrm{mmol}, 1.0$ equiv.) and alkyne ( $6.00 \mathrm{mmol}, 1.5$ equiv.) in acetone $(4.0 \mathrm{~mL})$. The solution was stirred at $55^{\circ} \mathrm{C}$ for $18 \mathrm{~h}$, after which time the reaction mixture was concentrated in vacuo. The crude material was purified by flash column chromatography.

\subsection{3. (E)-1-(2-(Phenylamino)cyclohex-1-en-1-yl)non-2-en-1- one, $(2 a)$}

Yellow oil, 73\% yield. $\mathrm{R}_{f} 0.28$ (petroleum ether / $\mathrm{Et}_{2} \mathrm{O}(95: 5)$ ); IR (thin film, $v_{\max } / \mathrm{cm}^{-1}$ ) 2927, 2856, 1575, 1499, 1206, 1169, 751, 698; ${ }^{1} \mathrm{H}$ NMR $\left(400 \mathrm{MHz}, \mathrm{CDCl}_{3}\right) \delta_{\mathrm{H}} 13.75(1 \mathrm{H}, \mathrm{s}), 7.31(2 \mathrm{H}, \mathrm{d}, J$ $=8.0 \mathrm{~Hz}), 7.14(1 \mathrm{H}, \mathrm{t}, J=7.5 \mathrm{~Hz}), 7.10(2 \mathrm{H}, \mathrm{d}, J=7.5 \mathrm{~Hz}), 6.85$ $(1 \mathrm{H}, \mathrm{dt}, J=15.0$ and $7.0 \mathrm{~Hz}), 6.58(1 \mathrm{H}, \mathrm{d}, J=15.0 \mathrm{~Hz}), 2.55(2 \mathrm{H}$, $\mathrm{t}, J=6.5 \mathrm{~Hz}), 2.42(2 \mathrm{H}, \mathrm{t}, J=6.0 \mathrm{~Hz}), 2.23(2 \mathrm{H}, \mathrm{qd}, J=7.0$ and $1.0 \mathrm{~Hz}), 1.72-1.66(2 \mathrm{H}, \mathrm{m}), 1.62-1.56(2 \mathrm{H}, \mathrm{m}), 1.51-1.43(2 \mathrm{H}, \mathrm{m})$, 1.37-1.23 (6H, m), $0.89(3 \mathrm{H}, \mathrm{t}, J=7.0 \mathrm{~Hz}) ;{ }^{13} \mathrm{C} \mathrm{NMR}(101 \mathrm{MHz}$, $\left.\mathrm{CDCl}_{3}\right) \delta_{\mathrm{C}} 188.4,160.8,144.2,139.3,129.0,127.2,125.4,125.1$, 102.1, 32.9, 31.8, 29.1, 28.8, 28.6, 25.5, 23.2, 22.7, 21.9, 14.2; HRMS $\left(\mathrm{ESI}^{+}\right)$calc. for $\mathrm{C}_{21} \mathrm{H}_{30} \mathrm{NO}[\mathrm{M}+\mathrm{H}]^{+}$312.2322, found 312.2321 .

\subsection{4. (E)-3-Cyclohexyl-1-(2-(phenylamino)cyclohex-1-en-1- yl)prop-2-en-1-one, (2b)}

Yellow oil, 77\% yield. $\mathrm{R}_{f} 0.24$ (petroleum ether / $\mathrm{Et}_{2} \mathrm{O}(95: 5)$ ); IR (thin film, $v_{\max } / \mathrm{cm}^{-1}$ ) 2923, 2850, 1572, 1499, 1163, 963, 752, 697; ${ }^{1} \mathrm{H}$ NMR $\left(400 \mathrm{MHz}, \mathrm{CDCl}_{3}\right) \delta_{\mathrm{H}} 13.78(1 \mathrm{H}, \mathrm{s}), 7.30(2 \mathrm{H}, \mathrm{t}, J$ $=8.0 \mathrm{~Hz}), 7.14(1 \mathrm{H}, \mathrm{t}, J=7.5 \mathrm{~Hz}), 7.09(2 \mathrm{H}, \mathrm{d}, J=7.5 \mathrm{~Hz}), 6.81$ $(1 \mathrm{H}, \mathrm{dd}, J=15.5$ and $7.0 \mathrm{~Hz}), 6.54(1 \mathrm{H}, \mathrm{dd}, J=15.5$ and $1.0 \mathrm{~Hz})$, $2.54(2 \mathrm{H}, \mathrm{t}, J=6.5 \mathrm{~Hz}), 2.41(2 \mathrm{H}, \mathrm{t}, J=6.0 \mathrm{~Hz}), 2.20-2.14(1 \mathrm{H}$, $\mathrm{m}), 1.80-1.66(7 \mathrm{H}, \mathrm{m}), 1.61-1.55(2 \mathrm{H}, \mathrm{m}), 1.36-1.13(5 \mathrm{H}, \mathrm{m}) ;{ }^{13} \mathrm{C}$ NMR $\left(101 \mathrm{MHz}, \mathrm{CDCl}_{3}\right) \delta_{\mathrm{C}} 188.6,160.7,149.2,139.3,129.0$, 125.4, 125.1, 124.6, 102.3, 41.1, 32.4, 28.6, 26.2, 26.0, 25.5, 23.2, 21.9; HRMS $\left(\mathrm{ESI}^{+}\right)$calc. for $\mathrm{C}_{21} \mathrm{H}_{28} \mathrm{NO}[\mathrm{M}+\mathrm{H}]^{+} 310.2165$, found 310.2165 .

\subsection{5. (E)-3-Cyclopentyl-1-(2-(phenylamino)cyclohex-1-en-1- yl)prop-2-en-1-one, (2c)}

Yellow oil, 53\%. $\mathrm{R}_{f} 0.33$ (petroleum ether / $\mathrm{Et}_{2} \mathrm{O}$ (90:10)); IR (thin film, $v_{\max } / \mathrm{cm}^{-1}$ ) 2939, 1574, 1295, 1254, 1208, 698; ${ }^{1} \mathrm{H}$ NMR $\left(400 \mathrm{MHz}, \mathrm{CDCl}_{3}\right) \delta_{\mathrm{H}} 13.78(1 \mathrm{H}, \mathrm{s}), 7.30(2 \mathrm{H}, \mathrm{t}, J=8.0 \mathrm{~Hz}), 7.15-$ $7.08(3 \mathrm{H}, \mathrm{m}), 6.84(1 \mathrm{H}, \mathrm{dd}, J=15.0$ and $8.5 \mathrm{~Hz}), 6.56(1 \mathrm{H}, \mathrm{d}, J=$ $15.0 \mathrm{~Hz}), 2.68-2.58(1 \mathrm{H}, \mathrm{m}), 2.54(2 \mathrm{H}, \mathrm{t}, J=6.5 \mathrm{~Hz}), 2.41(2 \mathrm{H}, \mathrm{t}$, 
$J=6.5 \mathrm{~Hz}), 1.87-1.80(2 \mathrm{H}, \mathrm{m}), 1.74-1.64(4 \mathrm{H}, \mathrm{m}), 1.63-1.55(4 \mathrm{H}$, m), 1.48-1.39 (2H, m); ${ }^{13} \mathrm{C}$ NMR (101 MHz, $\left.\mathrm{CDCl}_{3}\right) \delta_{\mathrm{C}} 188.4$, 160.7, 148.4, 139.3, 129.0, 125.3, 125.2, 125.1, 102.2, 43.5, 33.0, 28.6, 25.5, 25.4, 23.2, 21.9; HRMS (ESI ${ }^{+}$) calc. for $\mathrm{C}_{20} \mathrm{H}_{26} \mathrm{NO}$ $[\mathrm{M}+\mathrm{H}]^{+}$296.2009, found 296.2022.

\subsection{6. (E)-4,4-Dimethyl-1-(2-(phenylamino)cyclohex-1-en-1- yl)pent-2-en-1-one, $(\mathbf{2 d})$}

Yellow oil, 85\% yield. $\mathrm{R}_{f} 0.30$ (petroleum ether / $\mathrm{Et}_{2} \mathrm{O}(95: 5)$ ); IR (thin film, $v_{\max } / \mathrm{cm}^{-1}$ ) 2958, 1576, 1499, 1301, 1270, 1215, $1168 ;{ }^{1} \mathrm{H}$ NMR $\left(400 \mathrm{MHz}, \mathrm{CDCl}_{3}\right) \delta_{\mathrm{H}} 13.79(1 \mathrm{H}, \mathrm{s}), 7.31(2 \mathrm{H}, \mathrm{t}, J$ $=8.0 \mathrm{~Hz}), 7.14(1 \mathrm{H}, \mathrm{t}, J=7.5 \mathrm{~Hz}), 7.10(2 \mathrm{H}, \mathrm{d}, J=7.5 \mathrm{~Hz}), 6.87$ $(1 \mathrm{H}, \mathrm{d}, J=15.5 \mathrm{~Hz}), 6.49(1 \mathrm{H}, \mathrm{d}, J=15.5 \mathrm{~Hz}), 2.56(2 \mathrm{H}, \mathrm{t}, J=6.5$ $\mathrm{Hz}), 2.42(2 \mathrm{H}, \mathrm{t}, J=6.0 \mathrm{~Hz}), 1.73-1.66(2 \mathrm{H}, \mathrm{m}), 1.63-1.56(2 \mathrm{H}$, $\mathrm{m}), 1.11(9 \mathrm{H}, \mathrm{s}) ;{ }^{13} \mathrm{C}$ NMR $\left(101 \mathrm{MHz}, \mathrm{CDCl}_{3}\right) \delta_{\mathrm{C}} 188.7,160.7$, 154.1, 139.3, 129.0, 125.4, 125.1, 122.1, 102.4, 33.9, 29.2, 28.6, 25.5, 23.2, 21.9; HRMS $\left(\mathrm{ESI}^{+}\right)$calc. for $\mathrm{C}_{19} \mathrm{H}_{26} \mathrm{NO}[\mathrm{M}+\mathrm{H}]^{+}$ 284.2009, found 284.2007 .

\subsection{7. (E)-6-Chloro-1-(2-(phenylamino)cyclohex-1-en-1-yl)hex-2-} en-1-one, $(2 e)$

Yellow oil, 63\% yield. $\mathrm{R}_{f} 0.28$ (petroleum ether / $\mathrm{Et}_{2} \mathrm{O}(90: 10)$ ); IR (thin film, $v_{\max } / \mathrm{cm}^{-1}$ ) 2935, 1572, 1498, 1434, 1293, 1264, $1213,1179,750,698 ;{ }^{1} \mathrm{H}$ NMR $\left(400 \mathrm{MHz}, \mathrm{CDCl}_{3}\right) \delta_{\mathrm{H}} 13.77(1 \mathrm{H}$, s), $7.31(2 \mathrm{H}, \mathrm{t}, J=8.0 \mathrm{~Hz}), 7.16(1 \mathrm{H}, \mathrm{t}, J=7.5 \mathrm{~Hz}), 7.10(2 \mathrm{H}, \mathrm{d}, J$ $=7.5 \mathrm{~Hz}), 6.78(1 \mathrm{H}, \mathrm{dt}, J=15.0$ and $7.0 \mathrm{~Hz}), 6.64(1 \mathrm{H}, \mathrm{d}, J=15.0$ $\mathrm{Hz}), 3.57(2 \mathrm{H}, \mathrm{t}, J=6.5 \mathrm{~Hz}), 2.54(2 \mathrm{H}, \mathrm{t}, J=6.5 \mathrm{~Hz}), 2.43-2.38$ $(4 \mathrm{H}, \mathrm{m}), 1.99-1.92(2 \mathrm{H}, \mathrm{m}), 1.72-1.66(2 \mathrm{H}, \mathrm{m}), 1.62-1.56(2 \mathrm{H}, \mathrm{m})$; ${ }^{13} \mathrm{C}$ NMR $\left(101 \mathrm{MHz}, \mathrm{CDCl}_{3}\right) \delta_{\mathrm{C}} 187.7,161.3,141.3,139.1,129.0$, 128.5, 125.5, 125.3, 102.0, 44.4, 31.4, 29.8, 28.6, 25.4, 23.2, 21.8; HRMS $\left(\mathrm{ESI}^{+}\right)$calc. for $\mathrm{C} 18 \mathrm{H} 23^{35} \mathrm{CINO}[\mathrm{M}+\mathrm{H}]^{+} 304.1463$, found 304.1463 .

\subsection{8. (E)-6-((tert-Butyldimethylsilyl)oxy)-1-(2- (phenylamino)cyclohex-1-en-1-yl)hex-2-en-1-one, (2f)}

Yellow oil, 83\% yield. $\mathrm{R}_{f} 0.33$ (petroleum ether / $\mathrm{Et}_{2} \mathrm{O}$ (90:10)); IR (thin film, $v_{\max } / \mathrm{cm}^{-1}$ ) 2929, 2856, 1573, 1499, 1210, 1167, 1097,$834 ;{ }^{1} \mathrm{H}$ NMR $\left(400 \mathrm{MHz}, \mathrm{CDCl}_{3}\right) \delta_{\mathrm{H}} 13.76(1 \mathrm{H}, \mathrm{s}), 7.30(2 \mathrm{H}$, $\mathrm{t}, J=8.0 \mathrm{~Hz}), 7.14(1 \mathrm{H}, \mathrm{t}, J=7.5 \mathrm{~Hz}), 7.10(2 \mathrm{H}, \mathrm{d}, J=8.0 \mathrm{~Hz})$, $6.85(1 \mathrm{H}, \mathrm{dt}, J=15.0$ and $7.0 \mathrm{~Hz}), 6.60(1 \mathrm{H}, \mathrm{d}, J=15.0 \mathrm{~Hz}), 3.65$ $(2 \mathrm{H}, \mathrm{t}, J=6.0 \mathrm{~Hz}), 2.54(2 \mathrm{H}, \mathrm{t}, J=6.5 \mathrm{~Hz}), 2.41(2 \mathrm{H}, \mathrm{t}, J=6.5$ $\mathrm{Hz}), 2.30(2 \mathrm{H}, \mathrm{q}, J=7.0 \mathrm{~Hz}), 1.73-1.66(4 \mathrm{H}, \mathrm{m}), 1.61-1.55(2 \mathrm{H}$, m), $0.90(9 \mathrm{H}, \mathrm{s}), 0.06(6 \mathrm{H}, \mathrm{s}) ;{ }^{13} \mathrm{C}$ NMR $\left(101 \mathrm{MHz}, \mathrm{CDCl}_{3}\right) \delta_{\mathrm{C}}$ 188.1, 160.9, 143.4, 139.2, 129.0, 127.4, 125.4, 125.1, 102.0, 62.5, $31.8,29.2,28.6,26.1,25.5,23.2,21.9,18.4,-5.2$; HRMS (ESI $\left.{ }^{+}\right)$ calc. for $\mathrm{C}_{24} \mathrm{H}_{38} \mathrm{NO}_{2} \mathrm{Si}[\mathrm{M}+\mathrm{H}]^{+} 400.2666$, found 400.2676 .

\subsection{9. (E)-5-Phenyl-1-(2-(phenylamino)cyclohex-1-en-1-yl)pent- 2-en-1-one, (2g)}

Yellow oil, 46\%. $\mathrm{R}_{f} 0.18$ (petroleum ether $/ \mathrm{Et}_{2} \mathrm{O}(90: 10)$ ); IR (thin film, $\left.v_{\max } / \mathrm{cm}^{-1}\right) 2955,1573,1207,1171,689 ;{ }^{1} \mathrm{H}$ NMR (400 $\left.\mathrm{MHz} \mathrm{CDCl}_{3}\right) \delta_{\mathrm{H}} 13.75(1 \mathrm{H}, \mathrm{s}), 7.34-7.28(4 \mathrm{H}, \mathrm{m}), 7.23-7.19(3 \mathrm{H}$, m), $7.16(1 \mathrm{H}, \mathrm{t}, J=7.5 \mathrm{~Hz}), 7.11(2 \mathrm{H}, \mathrm{d}, J=8.0 \mathrm{~Hz}), 6.90(1 \mathrm{H}, \mathrm{dt}$, $J=15.0$ and $7.0 \mathrm{~Hz}), 6.60(1 \mathrm{H}, \mathrm{d}, J=15.0 \mathrm{~Hz}), 2.81(2 \mathrm{H}, \mathrm{dd}, J=$ 8.0 and $7.5 \mathrm{~Hz}), 2.57(2 \mathrm{H}, \mathrm{q}, J=7.0 \mathrm{~Hz}), 2.50(2 \mathrm{H}, \mathrm{t}, J=6.5 \mathrm{~Hz})$, $2.42(2 \mathrm{H}, \mathrm{t}, J=6.5 \mathrm{~Hz}), 1.72-1.66(2 \mathrm{H}, \mathrm{m}), 1.62-1.56(2 \mathrm{H}, \mathrm{m}) ;{ }^{13} \mathrm{C}$ NMR $\left(101 \mathrm{MHz}, \mathrm{CDCl}_{3}\right) \delta_{\mathrm{C}} 188.1,161.0,142.6,141.5,139.3$, $129.0,128.5,128.5,127.9,126.1,125.5,125.2,102.1,35.1,34.7$, 28.6, 25.5, 23.2, 21.9; HRMS (ESI ${ }^{+}$) calc. for $\mathrm{C}_{23} \mathrm{H}_{26} \mathrm{NO}[\mathrm{M}+\mathrm{H}]^{+}$ 332.2009 , found 332.2004.

\subsubsection{0. (E)-4-Cyclohexyl-1-(2-(phenylamino)cyclohex-1-en-1- yl)but-2-en-1-one, (2h)}

Yellow oil, 62\% yield. $\mathrm{R}_{f} 0.37$ (petroleum ether / $\mathrm{Et}_{2} \mathrm{O}(90: 10)$ ); IR (thin film, $v_{\max } / \mathrm{cm}^{-1}$ ) 2921, 2850, 1573, 1499, 1435, 1266,
1214, 1167; ${ }^{1} \mathrm{H}$ NMR (400 MHz, $\left.\mathrm{CDCl}_{3}\right) \delta_{\mathrm{H}} 13.76(1 \mathrm{H}, \mathrm{s}), 7.31$ $(2 \mathrm{H}, \mathrm{t}, J=8.0 \mathrm{~Hz}), 7.14(1 \mathrm{H}, \mathrm{t}, J=8.0 \mathrm{~Hz}), 7.10(2 \mathrm{H}, \mathrm{d}, J=8.0$ $\mathrm{Hz}), 6.84(1 \mathrm{H}, \mathrm{dt}, J=15.0$ and $7.5 \mathrm{~Hz}), 6.56(1 \mathrm{H}, \mathrm{d}, J=15.0 \mathrm{~Hz})$, $2.54(2 \mathrm{H}, \mathrm{t}, J=6.5 \mathrm{~Hz}), 2.42(2 \mathrm{H}, \mathrm{t}, J=6.5 \mathrm{~Hz}), 2.13(2 \mathrm{H}, \mathrm{t}, J=$ $7.5 \mathrm{~Hz}), 1.77-1.56(9 \mathrm{H}, \mathrm{m}), 1.49-1.37(1 \mathrm{H}, \mathrm{m}), 1.28-1.10(3 \mathrm{H}, \mathrm{m})$, $0.95(2 \mathrm{H}, \mathrm{m}) ;{ }^{13} \mathrm{C}$ NMR $\left(101 \mathrm{MHz}, \mathrm{CDCl}_{3}\right) \delta_{\mathrm{C}} 188.2,160.8,142.9$, 139.3, 129.0, 128.2, 125.4, 125.1, 102.1, 40.9, 37.8, 33.4, 28.6, 26.6, 26.4, 25.5, 23.2, 21.9; HRMS $\left(\mathrm{ESI}^{+}\right)$calc. for $\mathrm{C}_{22} \mathrm{H}_{30} \mathrm{NO}$ $[\mathrm{M}+\mathrm{H}]^{+}$324.2322, found 324.2321.

\subsubsection{1. (E)-2-(7-Oxo-7-(2-(phenylamino)cyclohex-1-en-1-yl)hept- 5-en-1-yl)isoindoline-1,3-dione, (2i)}

Yellow oil, 50\% yield. $\mathrm{R}_{f} 0.26$ (petroleum ether / EtOAc (80:20)); IR (thin film, $v_{\max } / \mathrm{cm}^{-1}$ ) 2980, 2936, 1711, 1574, 1396, 1170,$720 ;{ }^{1} \mathrm{H}$ NMR $\left(400 \mathrm{MHz}, \mathrm{CDCl}_{3}\right) \delta_{\mathrm{H}} 13.73(1 \mathrm{H}, \mathrm{s}), 7.84-$ $7.79(2 \mathrm{H}, \mathrm{m}), 7.71-7.67(2 \mathrm{H}, \mathrm{m}), 7.29(2 \mathrm{H}, \mathrm{d}, J=8.0 \mathrm{~Hz}), 7.14-$ $7.07(3 \mathrm{H}, \mathrm{m}), 6.78(1 \mathrm{H}, \mathrm{dt}, J=15.0$ and $7.0 \mathrm{~Hz}), 6.57(1 \mathrm{H}, \mathrm{d}, J=$ $15.0 \mathrm{~Hz}), 3.69(2 \mathrm{H}, \mathrm{t}, J=7.5 \mathrm{~Hz}), 2.51(2 \mathrm{H}, \mathrm{t}, J=6.0 \mathrm{~Hz}), 2.39$ $(2 \mathrm{H}, \mathrm{t}, J=6.0 \mathrm{~Hz}), 2.28(2 \mathrm{H}, \mathrm{q}, J=7.0 \mathrm{~Hz}), 1.76-1.63(4 \mathrm{H}, \mathrm{m})$, 1.59-1.49 (4H, m); ${ }^{13} \mathrm{C}$ NMR $\left(101 \mathrm{MHz}, \mathrm{CDCl}_{3}\right) \delta_{\mathrm{C}} 188.0,168.4$, $160.9,143.0,139.2,133.9,132.2,129.0,127.7,125.4,125.1$, 123.2, 102.1, 37.8, 32.3, 28.5, 28.2, 26.0, 25.4, 23.2, 21.8; HRMS $\left(\mathrm{ESI}^{+}\right.$) calc. for $\mathrm{C}_{27} \mathrm{H}_{29} \mathrm{~N}_{2} \mathrm{O}_{3}[\mathrm{M}+\mathrm{H}]^{+} 429.2173$, found 429.2162.

\subsubsection{2. (E)-1-(5-Methoxy-2-(phenylamino)cyclohex-1-en-1- yl)non-2-en-1-one, $(2 \mathrm{j})$}

Yellow oil, 71\% yield. $\mathrm{R}_{f} 0.29$ (petroleum ether / EtOAc (90:10)); IR (thin film, $v_{\max } / \mathrm{cm}^{-1}$ ) 2926, 1576, 1499, 1203, 1102, 780, 759, 694; ${ }^{1} \mathrm{H}$ NMR $\left(400 \mathrm{MHz}, \mathrm{CDCl}_{3}\right) \delta_{\mathrm{H}} 13.68(1 \mathrm{H}, \mathrm{s}), 7.31$ $(2 \mathrm{H}, \mathrm{t}, J=8.0 \mathrm{~Hz}), 7.16(1 \mathrm{H}, \mathrm{t}, J=8.0 \mathrm{~Hz}), 7.10(2 \mathrm{H}, \mathrm{d}, J=8.0$ $\mathrm{Hz}), 6.86(1 \mathrm{H}, \mathrm{dt}, J=15.0$ and $7.0 \mathrm{~Hz}), 6.55(1 \mathrm{H}, \mathrm{dt}, J=15.0$ and $1.5 \mathrm{~Hz}), 3.58-3.52(1 \mathrm{H}, \mathrm{m}), 3.41(3 \mathrm{H}, \mathrm{s}), 2.86(1 \mathrm{H}, \mathrm{dd}, J=15.0$ and $5.0 \mathrm{~Hz}), 2.62-2.52(2 \mathrm{H}, \mathrm{m}), 2.45-2.37(1 \mathrm{H}, \mathrm{m}), 2.23(2 \mathrm{H}, \mathrm{qd}$, $J=7.0$ and $1.5 \mathrm{~Hz}), 1.88-1.81(1 \mathrm{H}, \mathrm{m}), 1.70-1.62(1 \mathrm{H}, \mathrm{m}), 1.50-$ $1.43(2 \mathrm{H}, \mathrm{m}), 1.37-1.24(6 \mathrm{H}, \mathrm{m}), 0.89(3 \mathrm{H}, \mathrm{s}) ;{ }^{13} \mathrm{C}$ NMR $(101$ $\left.\mathrm{MHz}, \mathrm{CDCl}_{3}\right) \delta_{\mathrm{C}} 188.3,159.8,144.7,139.2,129.1,127.0,125.4$, $125.3,99.3,75.3,56.1,32.9,31.8,31.3,29.0,28.7,26.0,25.5$, 22.7, 14.2; HRMS (ESI ${ }^{+}$) calc. for $\mathrm{C}_{22} \mathrm{H}_{32} \mathrm{NO}_{2}[\mathrm{M}+\mathrm{H}]^{+}$342.2428, found 342.2435 .

\subsubsection{3. (E)-1-(4-(Phenylamino)-1,2,5,6-tetrahydro-[1,1'- biphenyl]-3-yl)non-2-en-1-one, (2k)}

Yellow oil, 56\% yield. $\mathrm{R}_{f} 0.28$ (petroleum ether / $\mathrm{Et}_{2} \mathrm{O}(90: 10)$ ); IR (thin film, $v_{\max } / \mathrm{cm}^{-1}$ ) 2980, 2927, 2349, 1573, 672, 665; ${ }^{1} \mathrm{H}$ NMR (400 MHz, $\left.\mathrm{CDCl}_{3}\right) \delta_{\mathrm{H}} 13.77(1 \mathrm{H}, \mathrm{s}), 7.37-7.23(7 \mathrm{H}, \mathrm{m})$, 7.19-7.13 (3H, m), $6.88(1 \mathrm{H}, \mathrm{dt}, J=15.0$ and $7.0 \mathrm{~Hz}), 6.55(1 \mathrm{H}, \mathrm{d}$, $J=15.0 \mathrm{~Hz}), 2.94-2.80(2 \mathrm{H}, \mathrm{m}), 2.67-2.52(3 \mathrm{H}, \mathrm{m}), 2.20(2 \mathrm{H}, \mathrm{q}, J$ $=7.0 \mathrm{~Hz}), 2.00-1.93(1 \mathrm{H}, \mathrm{m}), 1.82-1.71(1 \mathrm{H}, \mathrm{m}), 1.48-1.41(2 \mathrm{H}$, $\mathrm{m}), 1.35-1.22(6 \mathrm{H}, \mathrm{m}), 0.87(3 \mathrm{H}, \mathrm{t}, J=7.0 \mathrm{~Hz}) ;{ }^{13} \mathrm{C}$ NMR $(101$ $\left.\mathrm{MHz}, \mathrm{CDCl}_{3}\right) \delta_{\mathrm{C}} 188.2,160.1,146.4,144.7,139.3,129.1,128.6$, 127.1, 127.0, 126.5, 125.5, 125.3, 102.0, 40.9, 34.5, 32.9, 31.8, 29.0, 28.9, 28.7, 28.3, 22.7, 14.2; HRMS $\left(\mathrm{ESI}^{+}\right)$calc. for $\mathrm{C}_{27} \mathrm{H}_{34} \mathrm{NO}[\mathrm{M}+\mathrm{H}]^{+}$388.2635, found 388.2635.

\subsubsection{4. (E)-1-(5-(tert-Butyl)-2-(phenylamino)cyclohex-1-en-1- yl)non-2-en-1-one, (2l)}

Yellow oil, $43 \%$ yield. $\mathrm{R}_{f} 0.36$ (petroleum ether / $\mathrm{Et}_{2} \mathrm{O}(90: 10)$ ); IR (thin film, $v_{\max } / \mathrm{cm}^{-1}$ ) 2980, 2971, 1578, 1393, 1170, 967; ${ }^{1} \mathrm{H}$ NMR $\left(400 \mathrm{MHz}, \mathrm{CDCl}_{3}\right) \delta_{\mathrm{H}} 13.71(1 \mathrm{H}, \mathrm{s}), 7.30(2 \mathrm{H}, \mathrm{d}, J=7.5$ $\mathrm{Hz}), 7.16-7.08(3 \mathrm{H}, \mathrm{m}), 6.87(1 \mathrm{H}, \mathrm{dt}, J=15.5$ and $7.0 \mathrm{~Hz}), 6.59$ $(1 \mathrm{H}, \mathrm{d}, J=15.5 \mathrm{~Hz}), 2.62(1 \mathrm{H}, \mathrm{dd}, J=14.5$ and $5.0 \mathrm{~Hz}), 2.53-2.41$ (2H, m), 2.29-2.18 (3H, m), 1.85-1.78 (1H, m), 1.52-1.45 (2H, m), 1.39-1.26 (7H, m), 1.16-1.05 (1H, m), $0.94(9 \mathrm{H}, \mathrm{s}), 0.89(3 \mathrm{H}, \mathrm{t}, J$ $=7.0 \mathrm{~Hz}) ;{ }^{13} \mathrm{C}$ NMR $\left(101 \mathrm{MHz}, \mathrm{CDCl}_{3}\right) \delta_{\mathrm{C}} 188.5,160.6,144.3$, 139.4, 129.0, 127.1, 125.2, 125.0, 102.3, 44.8, 32.9, 32.6, 31.8, 
29.8, 29.1, 28.8, 27.4, 27.0, 23.1, 22.8, 14.2; HRMS (ESI $\left.{ }^{+}\right)$calc. for $\mathrm{C}_{25} \mathrm{H}_{38} \mathrm{NO}[\mathrm{M}+\mathrm{H}]^{+}$368.2948, found 368.2971.

\subsection{Tandem hydroacylation / enamine hydrolysis}

\subsubsection{General procedure}

Following completion of the $\beta$-aminoenal hydroacylation, $\mathrm{HCl}$ (aq. $2 \mathrm{M}, 0.6 \mathrm{~mL}$ ) was added and the reaction mixture stirred vigorously for a further $2 \mathrm{~h}$. Upon consumption of the hydroacylation product, as seen by TLC, the biphasic mixture was partitioned between water $(20 \mathrm{~mL})$ and $\mathrm{CH}_{2} \mathrm{Cl}_{2}(20 \mathrm{~mL})$. The aqueous layer was separated and extracted with $\mathrm{CH}_{2} \mathrm{Cl}_{2}(2 \times 20$ $\mathrm{mL})$. The combined organic extracts were dried over $\mathrm{MgSO}_{4}$, filtered, and concentrated in vacuo. The crude material was purified by flash column chromatography.

\subsection{2. (E)-2-(Non-2-enoyl)cyclohexan-1-one, (4a)}

Colourless oil, $56 \%$ yield. $\mathrm{R}_{f} 0.39$ (petroleum ether / $\mathrm{Et}_{2} \mathrm{O}$ (95:5)); IR (thin film, $v_{\max } / \mathrm{cm}^{-1}$ ) 2927, 2855, 1646, 1539, 1456, $1412,1304,990,669 ;{ }^{1} \mathrm{H}$ NMR $\left(400 \mathrm{MHz}, \mathrm{CDCl}_{3}\right) \delta_{\mathrm{H}} 16.75(1 \mathrm{H}$, s), $6.96(1 \mathrm{H}, \mathrm{dt}, J=15.5$ and $7.0 \mathrm{~Hz}), 6.30(1 \mathrm{H}, \mathrm{d}, J=15.5 \mathrm{~Hz})$, $2.43-2.36(4 \mathrm{H}, \mathrm{m}), 2.24(2 \mathrm{H}, \mathrm{qd}, J=7.5$ and $1.0 \mathrm{~Hz}), 1.74-1.65$ $(4 \mathrm{H}, \mathrm{m}), 1.49-1.42(2 \mathrm{H}, \mathrm{m}), 1.35-1.23(6 \mathrm{H}, \mathrm{m}), 0.87$ (3H, t, $J=7.0$ $\mathrm{Hz}) ;{ }^{13} \mathrm{C}$ NMR $\left(101 \mathrm{MHz}, \mathrm{CDCl}_{3}\right) \delta_{\mathrm{C}} 191.8,182.4,146.9,123.0$, 106.3, 33.8, 33.2, 31.8, 29.0, 28.5, 23.9, 23.1, 22.7, 21.9, 14.2; HRMS $\left(\mathrm{ESI}^{+}\right)$calc. for $\mathrm{C}_{15} \mathrm{H}_{25} \mathrm{O}_{2}[\mathrm{M}+\mathrm{H}]^{+}$237.1849, found 237.1810 .

\subsection{3. (E)-2-(3-Cyclohexylacryloyl)cyclohexan-1-one, (4b)}

Colourless oil, 66\% yield. $\mathrm{R}_{f} 0.35$ (petroleum ether / $\mathrm{Et}_{2} \mathrm{O}$ (95:5)); IR (thin film, $v_{\max } / \mathrm{cm}^{-1}$ ) 2924, 2851, 1640, 1559, 1447, $1410,1301,1168,963 ;{ }^{1} \mathrm{H}$ NMR $\left(400 \mathrm{MHz}, \mathrm{CDCl}_{3}\right) \delta_{\mathrm{H}} 16.75(1 \mathrm{H}$, s), $6.90(1 \mathrm{H}, \mathrm{dd}, J=15.5$ and $7.0 \mathrm{~Hz}), 6.25(1 \mathrm{H}, \mathrm{d}, J=15.5 \mathrm{~Hz})$, 2.43-2.40 (2H, m), 2.38-2.35 (2H, m), 2.21-2.12 (1H, m), 1.78$1.65(9 \mathrm{H}, \mathrm{m}), 1.35-1.11(5 \mathrm{H}, \mathrm{m}) ;{ }^{13} \mathrm{C}$ NMR $\left(101 \mathrm{MHz}, \mathrm{CDCl}_{3}\right) \delta_{\mathrm{C}}$ 191.6, 182.8, 151.7, 120.5, 106.5, 41.3, 33.7, 32.1, 26.1, 25.9, 23.9, 23.1, 21.9; HRMS (ESI $)$ calc. for $\mathrm{C}_{15} \mathrm{H}_{22} \mathrm{O}_{2}[\mathrm{M}+\mathrm{H}]^{+}$ 235.1693 , found 235.1701 .

\subsection{4. (E)-2-(4,4-Dimethylpent-2-enoyl)cyclohexan-1-one, (4d)}

Colourless oil, $88 \%$ yield. $\mathrm{R}_{f} 0.36$ (petroleum ether / $\mathrm{Et}_{2} \mathrm{O}$ (95:5)); IR (thin film, $v_{\max } / \mathrm{cm}^{-1}$ ) 2953, 2866, 1639, 1561, 1460, 1410, 1309, 1270, 1168, 979; ${ }^{1} \mathrm{H}$ NMR $\left(400 \mathrm{MHz}, \mathrm{CDCl}_{3}\right) \delta_{\mathrm{H}}$ $16.75(1 \mathrm{H}, \mathrm{s}), 6.96(1 \mathrm{H}, \mathrm{d}, J=15.5 \mathrm{~Hz}), 6.20(1 \mathrm{H}, \mathrm{d}, J=15.5 \mathrm{~Hz})$, 2.43-2.41 (2H, m), 2.38-2.35 (2H, m), 1.73-1.66 (4H, m), 1.08 $(9 \mathrm{H}, \mathrm{s}) ;{ }^{13} \mathrm{C} \mathrm{NMR}\left(101 \mathrm{MHz}, \mathrm{CDCl}_{3}\right) \delta_{\mathrm{C}} 191.3,183.1,156.4$, 118.1, 106.6, 34.2, 33.7, 28.9, 23.9, 23.1, 21.8; HRMS $\left(\mathrm{ESI}^{+}\right)$calc. for $\mathrm{C}_{13} \mathrm{H}_{21} \mathrm{O}_{2}[\mathrm{M}+\mathrm{H}]^{+}$209.1536, found 209.1548.

\subsection{5. (E)-2-(6-Chlorohex-2-enoyl)cyclohexan-1-one, (4e)}

Colourless oil, $70 \%$ yield. $\mathrm{R}_{f} 0.17$ (petroleum ether / $\mathrm{Et}_{2} \mathrm{O}$ (95:5)); IR (thin film, $v_{\max } / \mathrm{cm}^{-1}$ ) 2938, 2865, 1712, 1645, 1561, 1446, 1410, 1299, 1168, 969; ${ }^{1} \mathrm{H}$ NMR $\left(400 \mathrm{MHz}, \mathrm{CDCl}_{3}\right) \delta_{\mathrm{H}}$ $16.65(1 \mathrm{H}, \mathrm{s}), 6.88(1 \mathrm{H}, \mathrm{dt}, J=15.5 \mathrm{and} 7.0 \mathrm{~Hz}), 6.36(1 \mathrm{H}, \mathrm{d}, J=$ $15.5 \mathrm{~Hz}), 3.55(2 \mathrm{H}, \mathrm{t}, J=6.5 \mathrm{~Hz}), 2.45-2.35(6 \mathrm{H}, \mathrm{m}), 1.98-1.91$ $(2 \mathrm{H}, \mathrm{m}), 1.74-1.66(4 \mathrm{H}, \mathrm{m}) ;{ }^{13} \mathrm{C}$ NMR $\left(101 \mathrm{MHz}, \mathrm{CDCl}_{3}\right) \delta_{\mathrm{C}}$ 192.6, 181.4, 143.9, 124.2, 106.5, 44.2, 33.9, 31.1, 30.1, 23.8, 23.1, 21.8; HRMS $\left(\mathrm{ESI}^{+}\right)$calc. for $\mathrm{C}_{12} \mathrm{H}_{18}{ }^{15} \mathrm{ClO}_{2}[\mathrm{M}+\mathrm{H}]^{+}$ 229.0990 , found 229.0993 .

\subsection{6. (E)-2-(5-Phenylpent-2-enoyl)cyclohexan-1-one, (4g)}

Colourless oil, $49 \%$ yield. $\mathrm{R}_{f} 0.27$ (petroleum ether $/ \mathrm{Et}_{2} \mathrm{O}$ (95:5)); IR (thin film, $v_{\max } / \mathrm{cm}^{-1}$ ) 3061, 3027, 2935, 2858, 1644, $1560,1496,1452,1409,1303,1168,967 ;{ }^{1} \mathrm{H}$ NMR $(400 \mathrm{MHz}$, $\left.\mathrm{CDCl}_{3}\right) \delta_{\mathrm{H}} 16.71(1 \mathrm{H}, \mathrm{s}), 7.30(2 \mathrm{H}, \mathrm{t}, J=8.0 \mathrm{~Hz}), 7.22-7.19(3 \mathrm{H}$, m), $7.00(1 \mathrm{H}, \mathrm{dt}, J=15.5$ and $7.0 \mathrm{~Hz}), 6.32(1 \mathrm{H}, \mathrm{d}, J=15.5 \mathrm{~Hz})$, $2.80(2 \mathrm{H}, \mathrm{t}, J=8.0 \mathrm{~Hz}), 2.58(2 \mathrm{H}, \mathrm{q}, J=7.5 \mathrm{~Hz}), 2.40-2.37(4 \mathrm{H}$, $\mathrm{m}), 1.75-1.66(4 \mathrm{H}, \mathrm{m}) ;{ }^{13} \mathrm{C} \mathrm{NMR}\left(101 \mathrm{MHz}, \mathrm{CDCl}_{3}\right) \delta_{\mathrm{C}} 192.2$, $181.8,145.1,141.1,128.6,128.5,126.2,123.6,106.4,34.8(2 \mathrm{C})$, 33.9, 23.8, 23.1, 21.8; HRMS $\left(\mathrm{ESI}^{+}\right)$calc. for $\mathrm{C}_{17} \mathrm{H}_{21} \mathrm{O}_{2}[\mathrm{M}+\mathrm{H}]^{+}$ 257.1536 , found 257.1542 .

\subsection{7. (E)-2-(4-cyclohexylbut-2-enoyl)cyclohexan-1-one, (4h)}

Colourless oil, $73 \%$ yield. $\mathrm{R}_{f} 0.45$ (petroleum ether / $\mathrm{Et}_{2} \mathrm{O}$ (90:10)); IR (thin film, $v_{\max } / \mathrm{cm}^{-1}$ ) 2922, 2850, 1643, 1562, 1447, $1410,1230,1168,1006,977 ;{ }^{1} \mathrm{H}$ NMR $\left(400 \mathrm{MHz}, \mathrm{CDCl}_{3}\right) \delta_{\mathrm{H}}$ $16.73(1 \mathrm{H}, \mathrm{s}), 6.94(1 \mathrm{H}, \mathrm{dt}, J=15.5 \mathrm{and} 7.5 \mathrm{~Hz}), 6.27(1 \mathrm{H}, \mathrm{d}, J=$ $15.5 \mathrm{~Hz}), 2.42-2.35(4 \mathrm{H}, \mathrm{m}), 2.13(2 \mathrm{H}, \mathrm{t}, J=7.0 \mathrm{~Hz}), 1.71-1.61$ $(9 \mathrm{H}, \mathrm{m}), 1.48-1.36(1 \mathrm{H}, \mathrm{m}), 1.27-1.08(3 \mathrm{H}, \mathrm{m}), 0.97-0.87(2 \mathrm{H}, \mathrm{m})$; ${ }^{13} \mathrm{C}$ NMR $\left(101 \mathrm{MHz}, \mathrm{CDCl}_{3}\right) \delta_{\mathrm{C}} 191.8,182.2,145.6,124.0,106.2$, 41.1, 37.7, 33.8, 33.3, 26.5, 26.3, 23.9, 23.1, 21.9; HRMS $\left(\mathrm{ESI}^{+}\right)$ calc. for $\mathrm{C}_{16} \mathrm{H}_{25} \mathrm{O}_{2}[\mathrm{M}+\mathrm{H}]^{+} 249.1849$, found 249.1849 .

\subsection{Aza-conjugate addition}

\subsubsection{General procedure}

To an argon-filled vial containing $\beta$-aminoenone $(0.200 \mathrm{mmol}$, 1.0 equiv.) and antimony(III) trichloride $(0.060 \mathrm{mmol}, 30 \mathrm{~mol} \%)$ was added $\mathrm{MeCN}(0.3 \mathrm{M})$, and the reaction mixture stirred for 18 hours at $55^{\circ} \mathrm{C}$. The reaction mixture was concentrated in vacuo and purified by flash column chromatography.

\subsubsection{2-Hexyl-1-phenyl-2,3,5,6,7,8-hexahydroquinolin-4(1H)- one, $(6 a)$}

Colourless oil, 73\%. $\mathrm{R}_{f} 0.18$ (petroleum ether / EtOAc (80:20)); IR (thin film, $v_{\max } / \mathrm{cm}^{-1}$ ) 2927, 2856, 1634, 1561, 1491, 1419, 1285, 1190, 1166; ${ }^{1} \mathrm{H}$ NMR (400 MHz, $\left.\mathrm{CDCl}_{3}\right) \delta_{\mathrm{H}} 7.38(2 \mathrm{H}, \mathrm{t}, J=$ $8.0 \mathrm{~Hz}), 7.29(1 \mathrm{H}, \mathrm{t}, J=7.5 \mathrm{~Hz}), 7.13(2 \mathrm{H}, \mathrm{t}, J=8.0 \mathrm{~Hz}), 3.72-$ $3.66(1 \mathrm{H}, \mathrm{m}), 2.84(1 \mathrm{H}, \mathrm{dd}, J=16.5$ and $6.0 \mathrm{~Hz}), 2.43(1 \mathrm{H}, \mathrm{dd}, J$ $=16.5$ and $6.0 \mathrm{~Hz}), 2.41-2.27(2 \mathrm{H}, \mathrm{m}), 2.00-1.85(2 \mathrm{H}, \mathrm{m}), 1.72-$ $1.46(6 \mathrm{H}, \mathrm{m}), 1.34-1.08(8 \mathrm{H}, \mathrm{m}), 0.82(3 \mathrm{H}, \mathrm{t}, J=7.0 \mathrm{~Hz}) ;{ }^{13} \mathrm{C} \mathrm{NMR}$ $\left(101 \mathrm{MHz}, \mathrm{CDCl}_{3}\right) \delta_{\mathrm{C}} 191.0,158.0,144.0,129.4,128.5,127.2$, $108.4,61.2,40.0,31.8,30.6,30.1,29.2,25.8,22.7,22.6,22.3$, 21.8, 14.1; HRMS $\left(\mathrm{ESI}^{+}\right)$calc. for $\mathrm{C}_{21} \mathrm{H}_{30} \mathrm{NO}[\mathrm{M}+\mathrm{H}]^{+} 312.2322$, found 312.2321 .

\subsubsection{2-Hexyl-1,6-diphenyl-2,3,5,6,7,8-hexahydroquinolin- 4(1H)-one, (6b)}

Colourless oil, isolated as an inseparable mixture of diastereomers $(1.4: 1 \mathrm{dr}) .85 \%$ yield. $\mathrm{R}_{f} 0.20$ (petroleum ether / EtOAc (80:20)); IR (thin film, $v_{\max } / \mathrm{cm}^{-1}$ ) 3028, 2926, 2856, 1632 1561, 1491, 1453, 1419, 1181; ${ }^{1} \mathrm{H}$ NMR $\left(400 \mathrm{MHz}, \mathrm{CDCl}_{3}\right) \delta_{\mathrm{H}}$ 7.35-7.30 (4H, m), 7.25-7.13 (6H, m), 7.25-7.13 (10H, m), 3.71$3.62(2 \mathrm{H}, \mathrm{m}), 2.91-2.61(6 \mathrm{H}, \mathrm{m}), 2.44-2.38(2 \mathrm{H}, \mathrm{m}), 2.32-2.11$ $(3 \mathrm{H}, \mathrm{m}), 2.05-1.91(3 \mathrm{H}, \mathrm{m}), 1.82-1.39(8 \mathrm{H}, \mathrm{m}), 1.26-1.02(16 \mathrm{H}$, $\mathrm{m}), 0.78-0.74(6 \mathrm{H}, \mathrm{m}) ;{ }^{13} \mathrm{C}$ NMR $\left(101 \mathrm{MHz}, \mathrm{CDCl}_{3}\right) \delta_{\mathrm{C}} 191.1$, $190.4,158.3,156.6,146.2,145.9,144.0,143.6,129.5,128.6$, $128.3,128.3,127.4,126.9,126.1,108.4,107.4,61.3,61.0,40.5$, $39.7,39.5,39.3,31.7,31.7,31.6,30.4,30.1,29.5_{3}, 29.5_{0}, 29.4_{2}$, $29.3_{7}, 29.2,25.8,25.5,22.6,14.1$; HRMS $\left(\mathrm{ESI}^{+}\right)$calc. for $\mathrm{C}_{27} \mathrm{H}_{34} \mathrm{NO}[\mathrm{M}+\mathrm{H}]^{+}$388.2635, found 388.2643.

\subsubsection{6-(tert-Butyl)-2-hexyl-1-phenyl-2,3,5,6, 7,8- hexahydroquinolin-4(1H)-one, (6c)}

Diastereomer 1: Colourless oil, $47 \%$ yield. $\mathrm{R}_{f} 0.40$ (petroleum ether / EtOAc (80:20)); IR (thin film, $v_{\max } / \mathrm{cm}^{-1}$ ) 2929, 2860, 1638, 1571, 1491, 1421, 1364, 1281, 1178; ${ }^{1} \mathrm{H}$ NMR (400 MHz, $\mathrm{CDCl}_{3}$ ) $\delta_{\mathrm{H}} 7.37(2 \mathrm{H}, \mathrm{d}, J=8.0 \mathrm{~Hz}), 7.28(1 \mathrm{H}, \mathrm{t}, J=8.0 \mathrm{~Hz}), 7.11(2 \mathrm{H}, \mathrm{d}, J$ $=8.0 \mathrm{~Hz}), 3.74(1 \mathrm{H}, \mathrm{m}), 2.71(1 \mathrm{H}, \mathrm{dd}, J=16.0$ and $5.0 \mathrm{~Hz}), 2.65-$ $2.60(1 \mathrm{H}, \mathrm{m}), 2.43(1 \mathrm{H}, \mathrm{dd}, J=16.0$ and $10.0 \mathrm{~Hz}), 2.02-1.86(3 \mathrm{H}$, 
m), 1.73-1.68 (1H, m), 1.55-1.39 (2H, m), 1,32-1.05 (10H, m), $0.87(9 \mathrm{H}, \mathrm{s}), 0.82(3 \mathrm{H}, \mathrm{t}, J=7.0 \mathrm{~Hz}) ;{ }^{13} \mathrm{C} \mathrm{NMR}\left(101 \mathrm{MHz}, \mathrm{CDCl}_{3}\right)$ $\delta_{\mathrm{C}} 191.8,159.4,143.7,129.4,128.2,127.2,109.4,60.9,44.2,40.9$, $32.5,32.2,31.8,31.6,29.2,27.4,25.5,24.1,23.4,22.6,14.1$; HRMS $\left(\mathrm{ESI}^{+}\right)$calc. for $\mathrm{C}_{25} \mathrm{H}_{38} \mathrm{NO}[\mathrm{M}+\mathrm{H}]^{+} 368.2948$, found 368.2957.

Diastereomer 2: Colourless oil, $31 \%$ yield. $\mathrm{R}_{f} 0.23$ (petroleum ether / EtOAc (80:20)); IR (thin film, $v_{\max } / \mathrm{cm}^{-1}$ ) 2930, 2858, 1633, $1565,1491,1420,1379,1232,1183 ;{ }^{1} \mathrm{H}$ NMR $\left(400 \mathrm{MHz}, \mathrm{CDCl}_{3}\right)$ $\delta_{\mathrm{H}} 7.39(2 \mathrm{H}, \mathrm{d}, J=8.0 \mathrm{~Hz}), 7.29(1 \mathrm{H}, \mathrm{t}, J=8.0 \mathrm{~Hz}), 7.14(2 \mathrm{H}, \mathrm{d}, J$ $=8.0 \mathrm{~Hz}), 3.71-3.65(1 \mathrm{H}, \mathrm{m}), 2.94(1 \mathrm{H}, \mathrm{dd}, J=16.5$ and $6.5 \mathrm{~Hz})$, $2.62(1 \mathrm{H}, \mathrm{dd}, J=16.0$ and $4.5 \mathrm{~Hz}), 2.46(1 \mathrm{H}, \mathrm{dd}, J=16.5$ and 3.5 $\mathrm{Hz}), 2.11-1.68(5 \mathrm{H}, \mathrm{m}), 1.60-1.52(1 \mathrm{H}, \mathrm{m}), 1.34-0.98(10 \mathrm{H}, \mathrm{m})$, $0.88(9 \mathrm{H}, \mathrm{s}), 0.82(3 \mathrm{H}, \mathrm{t}, J=7.0 \mathrm{~Hz}) ;{ }^{13} \mathrm{C} \mathrm{NMR}\left(101 \mathrm{MHz}, \mathrm{CDCl}_{3}\right)$ $\delta_{\mathrm{C}} 190.7,157.1,144.3,129.4,128.7,127.2,107.8,61.4,44.2,39.5$, $32.5,31.8,31.4,29.3,29.2,27.4,25.9,23.8,23.4,22.7,14.2$; HRMS $\left(\mathrm{ESI}^{+}\right.$) calc. for $\mathrm{C}_{25} \mathrm{H}_{38} \mathrm{NO}[\mathrm{M}+\mathrm{H}]^{+} 368.2948$, found 368.2956 .

\section{Acknowledgements}

We thank the EPSRC for financial support for this research.

\section{Appendix A. Supplementary data}

Supplementary data relating to this article may be found at.

\section{References}

1. Newhouse, T.; Baran, P. S.; Hoffmann, R. W. Chem. Soc. Rev. 2009, 38,3010 .

2. Trost, B. M. Science 1991, 254, 1471.

3. Moxham, G. L.; Randell-Sly, H.; Brayshaw, S. K.; Weller, A. S.; Willis, M. C. Chem. Eur. J. 2008, 14, 8383.

4. Leung, J. C.; Krische, M. J. Chem. Sci. 2012, 3, 2202.

5. Straker, R. N.; Majhail, M. K.; Willis, M. C. Chem. Sci. 2017, 8, 7963.

6. Majhail, M. K.; Ylioja, P. M.; Willis, M. C. Chem. Eur. J. 2016, 22, 7879 .

7. Neuhaus, J. D.; Morrow, S. M.; Brunavs, M.; Willis, M. C. Org. Lett. 2016, 18,1562

8. Marder, T. B.; Roe, D. C.; Milstein, D. Organometallics 1988, 7, 1451

9. Roy, A. H.; Lenges, C. P.; Brookhart, M. J. Am. Chem. Soc. 2007, 129, 2082.

10. Chen, Q. A.; Cruz, F. A.; Dong, V. M. J. Am. Chem. Soc. 2015, 137, 3157.

11. Kim, J.; Yi, C. S. ACS Catal. 2016, 6, 3336.

12. Omura, S.; Fukuyama, T.; Horiguchi, J.; Murakami, Y.; Ryu, I. J. Am Chem. Soc. 2008, 130, 14094

13. Shibahara, F.; Bower, J. F.; Krische, M. J. J. Am. Chem. Soc. 2008, 130, 14120.

14. Chen, Q. A.; Kim, D. K.; Dong, V. M. J. Am. Chem. Soc. 2014, 136, 3772 .

15. Lenges, C. P.; Brookhart, M. J. Am. Chem. Soc. 1997, 119, 3165.

16. Tsuda, T.; Kiyoi, T.; Saegusa, T. J. Org. Chem. 1990, 55, 2554.

17. Xiao, L. J.; Fu, X. N.; Zhou, M. J.; Xie, J. H.; Wang, L. X.; Xu, X. F.; Zhou, Q. L. J. Am. Chem. Soc. 2016, 138, 2957.

18. Kokubo, K.; Matsumasa, K.; Miura, M.; Nomura, M. J. Org. Chem. 1997, 62, 4564 .

19. Imai, M.; Tanaka, M.; Tanaka, K.; Yamamoto, Y.; Imai-Ogata, N.; Shimowatari, M.; Nagumo, S.; Kawahara, N.; Suemune, H. J. Org. Chem. 2004, 69, 1144

20. Murphy, S. K.; Petrone, D. A.; Coulter, M. M.; Dong, V. M. Org. Lett. 2011, 13, 6216 .

21. Murphy, S. K.; Coulter, M. M.; Dong, V. M. Chem. Sci. 2012, 3, 355

22. Willis, M. C.; McNally, S. J.; Beswick, P. J. Angew. Chem. Int. Ed. 2004, 43,340

23. Willis, M. C.; Woodward, R. L. J. Am. Chem. Soc. 2005, 127, 18012.

24. Willis, M. C.; Randell-Sly, H. E.; Woodward, R. L.; McNally, S. J.; Currie, G. S. J. Org. Chem. 2006, 71, 5291

25. Moxham, G. L.; Randell-Sly, H. E.; Brayshaw, S. K.; Woodward, R. L.; Weller, A. S.; Willis, M. C. Angew. Chem. Int. Ed. 2006, 45, 7618.

26. Osborne, J. D.; Willis, M. C. Chem. Commun. 2008, 40, 5025.
27. Stemmler, R. T.; Bolm, C. Adv. Synth. Catal. 2007, 349, 1185.

28. Coxon, T. J.; Fernández, M.; Barwick-Silk, J.; McKay, A. I.; Britton, L. E.; Weller, A. S.; Willis, M. C. J. Am. Chem. Soc. 2017, 139, 10142.

29. Lue, P.; Greenhill, J. V. Advances in Heterocyclic Chemistry, 1996, 67, 207.

30. Elassar, A.-Z. A.; El-Khair, A. A. Tetrahedron 2003, 59, 8463.

31. Negri, G.; Kascheres, C.; Kascheres, A. J. J. Heterocycl. Chem. 2004, 41,461 .

32. Michael, J. P.; de Koning, C. B.; Gravestock, D.; Hosken, G. D.; Howard, A. S.; Jungmann, C. M.; Krause, R. W. M.; Parsons, A. S.; Pelly, S. C.; Stanbury, T. V. Pure Appl. Chem. 1999, 71, 979.

33. Castaing, M.; Wason, S. L.; Estepa, B.; Hooper, J. F.; Willis, M. C. Angew. Chem. Int. Ed. 2013, 52, 13280.

34. Bhattacharya, R. N.; Kundu, P.; Maiti, G. Synth. Commun. 2010, 40, 476. 